\title{
Circulating adhesion molecules and subclinical interstitial lung disease: the Multi-Ethnic Study of Atherosclerosis
}

\author{
Claire F. McGroder ${ }^{1}$, Carrie P. Aaron ${ }^{2}$, Suzette J. Bielinski ${ }^{3}$, Steven M. Kawut ${ }^{4}$, \\ Russell P. Tracy ${ }^{5}$, Ganesh Raghu' ${ }^{6}$, R. Graham Barr ${ }^{1,7}$, David J. Lederer $\mathbb{1}^{1,7}$ and \\ Anna J. Podolanczuk $\mathbb{1}^{1}$ \\ Affiliations: ${ }^{1}$ Dept of Medicine, Columbia University Medical Center, New York, NY, USA. ${ }^{2}$ Dept of Medicine, \\ Brigham and Women's Hospital, Boston, MA, USA. ${ }^{3}$ Dept of Health Sciences Clinic, Mayo Clinic, Rochester, \\ MN, USA. ${ }^{4}$ Dept of Medicine and the Center for Clinical Epidemiology and Biostatistics, Perelman School of \\ Medicine at the University of Pennsylvania, Philadelphia, PA, USA. ${ }^{5}$ Dept of Pathology, University of Vermont, \\ Colchester, VT, USA. ${ }^{6}$ Dept of Medicine, University of Washington, Seattle, WA, USA. ${ }^{7}$ Dept of Epidemiology, \\ Columbia University Medical Center, New York, NY, USA.
}

Correspondence: Anna J. Podolanczuk, Columbia University Medical Center, 622 W 168th St, PH-8 East, New York, NY 10032, USA. E-mail: ajp2158वcumc.columbia.edu

@ERSpublications

In community-dwelling adults, adhesion molecules sICAM-1, sVCAM-1 and P-selectin are associated with CT measures of early lung injury, lower FVC, ILD hospitalisations and deaths, suggesting they may contribute to the development of pulmonary fibrosis http://bit.ly/2Xj0B6F

Cite this article as: McGroder CF, Aaron CP, Bielinski SJ, et al. Circulating adhesion molecules and subclinical interstitial lung disease: the Multi-Ethnic Study of Atherosclerosis. Eur Respir J 2019; 54: 1900295 [https://doi.org/10.1183/13993003.00295-2019].

ABSTRACT Adhesion molecules may contribute to the development of interstitial lung disease (ILD) and have been proposed as prognostic biomarkers in idiopathic pulmonary fibrosis. Our objective was to determine whether the circulating adhesion molecules soluble intracellular adhesion molecule (sICAM)-1, soluble vascular cell adhesion molecule (sVCAM)-1 and P-selectin are associated with subclinical ILD in community-dwelling adults.

The Multi-Ethnic Study of Atherosclerosis enrolled males and females aged 45-84 years from six communities in the United States in 2000-2002. High attenuation areas were defined as the percentage of imaged lung volume with attenuation $-600--250 \mathrm{HU}$ on cardiac computed tomography (CT). Interstitial lung abnormalities were visually assessed on full-lung CT. Spirometry was performed on a subset of individuals. ILD hospitalisations and deaths were adjudicated.

In fully adjusted analyses, higher levels of sICAM-1, sVCAM-1 and P-selectin were associated with greater high attenuation areas $(2.94 \%, 95 \%$ CI $1.80-4.07 \%$; $1.24 \%, 95 \%$ CI $0.14-2.35 \%$; and $1.58 \%$, 95\% CI $0.92-2.23 \%$, respectively), and greater rate of ILD hospitalisations (HR 1.36, 95\% CI 1.03-1.80; $1.40,95 \%$ CI 1.07-1.85; and 2.03, 95\% CI 1.16-3.5, respectively). sICAM-1 was associated with greater prevalence of interstitial lung abnormalities (OR 1.39, 95\% CI 1.13-1.71). sICAM-1 and P-selectin were associated with lower forced vital capacity $(44 \mathrm{~mL}, 95 \% \mathrm{CI} 12-76 \mathrm{~mL}$ and $29 \mathrm{~mL}, 95 \%$ CI 8-49 mL, respectively). sVCAM-1 and P-selectin were associated with increased risk of ILD death (HR 2.15, 95\% CI 1.26-3.64 and 3.61, 95\% CI 1.54-8.46, respectively).

Higher levels of circulating sICAM-1, sVCAM-1 and P-selectin are independently associated with CT and spirometric measures of subclinical ILD, and increased rate of adjudicated ILD events among community-dwelling adults. 


\section{Introduction}

Interstitial lung disease (ILD) is characterised by the presence of cellular proliferation, cellular infiltration and/or fibrosis of the lung parenchyma not due to infection or malignancy [1,2]. Cellular-level changes that lead to ILD probably begin years prior to the onset of symptoms [3]. Fibrotic ILDs generally have a poor prognosis and few treatment options [4]. The investigation of subclinical ILD has gained momentum in recent years as a way to identify novel risk factors and early mechanisms of lung fibrosis in humans [5-9]. These studies may lead to future interventions to prevent lung fibrosis [1].

Adhesion molecules mediate leukocyte recruitment into the lung, and may play a role in the development of ILD [10, 11]. Intracellular adhesion molecule (ICAM)-1 is overexpressed in idiopathic pulmonary fibrosis (IPF) lungs. Serum levels of soluble (s)ICAM-1 and soluble vascular cell adhesion molecule (sVCAM)-1 correlate with the extent of fibrosis and poor survival in IPF, and have been proposed as potential prognostic biomarkers $[10,12,13]$. The selectin family of adhesion molecules, including E-selectin and L-selectin, are an important part of the innate immune response [11, 14]. P-selectin has been implicated in lung injury, but has not been studied extensively in ILD [15]. The role of these adhesion molecules in adults with subclinical ILD is not known.

In the current study, we sought to determine the associations of circulating sICAM-1, sVCAM-1, E-selectin, L-selectin and P-selectin with computed tomography (CT) measures of subclinical ILD, forced vital capacity (FVC), and clinical ILD events in a large cohort of community-dwelling adults enrolled in the Multi-Ethnic Study of Atherosclerosis (MESA). We hypothesised that higher levels of circulating cell adhesion molecules would be associated with greater high attenuation areas (HAA), higher odds of interstitial lung abnormalities (ILA), lower FVC and increased risk of ILD events during follow-up.

\section{Methods}

Participants

MESA is a multicentre population-based prospective cohort study that enrolled 6814 males and females aged 45-84 years without evidence of clinical cardiovascular disease at study entry. MESA participants were sampled from six communities in the United States in the years 2000-2002, regardless of lung disease, respiratory symptoms or smoking history, as previously described [16]. Circulating adhesion molecules were measured in a random sample of MESA participants stratified by race/ethnicity. The sample sizes for each biomarker and timeline of measurements are shown in supplementary figure S1. Written informed consent was obtained from all participants and the study was approved by institutional review boards at all collaborating centres.

\section{Circulating adhesion molecules}

sICAM-1 and E-selectin were measured in blood samples collected at the MESA baseline exam in years 2000-2002. Samples were stored at $-80^{\circ} \mathrm{C}$ and analysed in the year 2004 after the first thaw. sICAM-1 was measured using ELISA (Parameter Human sICAM-1 Immunoassay; R\&D Systems, Minneapolis, MN, USA) in plasma from 2621 participants. E-selectin was measured using ELISA (Human Soluble E-Selectin kit; R\&D Systems) in serum from 999 participants. sVCAM-1, L-selectin and P-selectin were measured in blood samples collected at the MESA follow-up exam in years $2002-2004$. Samples were stored at $-80^{\circ} \mathrm{C}$, sent for processing after one thaw and analysed in years 2010-2011 after the second thaw cycle. Serum levels of sVCAM-1 and L-selectin were measured using ELISA (Quantikine Human Soluble VCAM Immunoassay kit, Human Soluble L-selectin Immunoassay kit; R\&D Systems) in 2440 participants. Plasma levels of P-selectin were measured in two independent batches from 6147 participants using ELISA (Human Soluble P-Selectin Immunoassay kit; R\&D Systems). Different sets of ELISA reagents and separate human control pool were used for each batch. The interassay coefficients of variation were $5.0 \%$, 3.6\%, 6.7\%, 7.3\% and 6.7\% for sICAM-1, sVCAM-1, P-selectin, E-selectin and L-selectin, respectively.

\section{Genotyping}

The sICAM-1 ELISAs can differ in their ability to recognise two of the less frequent sICAM-1 variants due to poor binding to the immunoassay [17]. Thus, the genotype of the sICAM-1 single nucleotide polymorphism (SNP) rs5491 was attained from all consenting individuals in order to ensure that the results were not affected by inclusion of individuals with the AT and TT variants. Genotyping was performed using the ITMAT-Broad-CARe (IBC) microarray (Illumina, San Diego, CA, USA).

\section{High attenuation areas on CT scan}

Lung attenuation was measured on exam 2 or 3 cardiac CT scans in 5703 MESA participants at the University of Iowa imaging lab using a modified version of Pulmonary Analysis Software Suite (University of Iowa, Iowa City, IA, USA). HAA was defined as percentage of imaged lung with attenuation between -600 and $-250 \mathrm{HU}$, as previously described $[5,18]$. Percentage emphysema was defined as the percentage of lung voxels below $-950 \mathrm{HU}$. 
Interstitial lung abnormalities on CT scan

Full-lung CT scans were performed at follow-up exam 5, in years 2010-2012, using multidetector CT scanners, as previously described [7]. 2907 participants had CT scans visually assessed by one of five expert radiologists (inter-reader $\kappa$ 0.47) for the presence of ILA, which was defined as the presence of ground-glass, reticular abnormalities, diffuse centrilobular nodularities, honeycombing, traction bronchiectasis, nonemphysematous cysts or architectural distortion in $\geqslant 5 \%$ of nondependent portions of the lung, as previously described $[7,19,20]$. Of these scans, 477 were classified as indeterminate for ILA and were excluded from ILA analyses.

\section{Spirometry}

Spirometry was performed at follow-up exams 3 and 4, between years 2004 and 2007, in accordance with American Thoracic Society/European Respiratory Society guidelines, using protocol that has been described previously $[21,22]$.

\section{Cause of death and hospitalisation}

Data on hospitalisations and deaths were obtained through direct contact with MESA participants or surrogates at 9-12-month intervals, and was supplemented by review of the National Death Index. Mortality data were complete as of March 2015 and hospitalisation data were complete as of December 2013. A two-member adjudication panel adjudicated all ILD deaths and hospitalisations, as described previously [9]. Respiratory death was determined based on the International Classification of Diseases-10 diagnosis code for underlying cause of death on the death certificate.

\section{Statistical analysis}

We examined the distributions of each biomarker using box plots and computed Pearson correlation coefficients between individual adhesion molecules. We used generalised linear models to examine the associations of circulating adhesion molecules, modelled in a continuous fashion, with HAA and FVC. We report all results per standard deviation of each adhesion molecule to facilitate interpretation. HAA was expressed as percentage of total imaged lung volume and was log-transformed. We used logistic regression to examine the association of adhesion molecules with ILA. HAA models were adjusted for age, sex, body mass index (BMI), waist circumference, height, race/ethnicity, current smoking status and pack-year smoking history, educational attainment, glomerular filtration rate, study site/scanner, radiation dose, percentage emphysema and total imaged lung volume. ILA and FVC models were adjusted for age, sex, race, smoking status, BMI and study site, as described previously [23]. We examined models stratified by SNP rs5491, smoking status, age and BMI, and used the likelihood ratio test to test for effect modification. To account for differences in P-selectin measurement between the two batches, we obtained residuals from a linear regression model using log-transformed raw P-selectin values as a response variable and batch number as a predictor, as previously described, and used the P-selectin residuals in our fully adjusted models [24]. We used Cox regression and proportional means models, which allow for recurrent events, to examine the associations of circulating adhesion molecules with time to ILD death and hospitalisation, respectively, as described previously [9]. Due to low event rates, we controlled for confounding in these models by calculating a generalised propensity score (GPS), as described previously [9]. We used generalised additive models with locally estimated scatterplot smoothers of continuous variables to examine for nonlinear associations between our predictors and outcomes, and to generate graphs. Statistical significance was defined using the Benjamini-Hochberg procedure to control the false discovery rate at 0.05. All analyses were performed in Stata (version 14; StataCorp, College Station, TX, USA) and R (version 3.5.1; www.r-project.org).

\section{Results}

The baseline characteristics of MESA participants overall and by quartile of each adhesion molecule are shown in table 1 and supplementary tables S1-S4. Overall, for participants with measured sICAM-1 and HAA, the mean \pm SD age was $59 \pm 9.6$ years. $56 \%$ of participants were female, $49 \%$ were white, $18 \%$ were black, $22 \%$ were Hispanic and $12 \%$ were Chinese. $45 \%$ were current or former smokers with a median 15 pack-years smoking history (table 1). Participants in the highest quartile of sICAM-1 were more likely to be female, white or Hispanic, ever-smokers and have higher BMI. The mean \pm SD levels of sICAM-1, sVCAM-1, P-selectin, E-selectin and L-selectin were $274 \pm 78.4 \mathrm{ng} \cdot \mathrm{mL}^{-1}, 740 \pm 232.0 \mathrm{ng} \cdot \mathrm{mL}^{-1}, 36 \pm 13.9 \mathrm{ng} \cdot \mathrm{mL}^{-1}, 55 \pm 25.3 \mathrm{ng} \cdot \mathrm{mL}^{-1}$ and $891 \pm 200.0 \mathrm{ng} \cdot \mathrm{mL}^{-1}$, respectively (supplementary figure S2). Pearson correlation coefficients demonstrated only weakly positive relationships between the adhesion molecules (supplementary table S5).

\section{High attenuation areas}

In fully adjusted models, higher levels of sICAM-1, sVCAM-1 and P-selectin were associated with greater HAA. Each standard-deviation increment of sICAM-1, sVCAM-1 and P-selectin was associated with a 
TABLE 1 Baseline characteristics of Multi-Ethnic Study of Atherosclerosis (MESA) participants by quartile of soluble intracellular adhesion molecule (sICAM)-1

\begin{tabular}{|c|c|c|c|c|c|}
\hline & \multirow[t]{2}{*}{ Overall } & \multicolumn{4}{|c|}{ Quartile of sICAM-1 } \\
\hline & & Q1 & Q2 & Q3 & Q4 \\
\hline Subjects $\mathrm{n}$ & 2233 & 559 & 558 & 558 & 558 \\
\hline $\mathrm{sICAM}-1 \mathrm{ng} \cdot \mathrm{mL}^{-1}$ & $273.8 \pm 78.39$ & $187.84 \pm 35.21$ & $248.43 \pm 11.02$ & $288.26 \pm 12.03$ & $373.29 \pm 70.38$ \\
\hline Age years & $59.1 \pm 9.6$ & $58.1 \pm 9.5$ & $59.8 \pm 9.8$ & $60.0 \pm 9.9$ & $60.0 \pm 9.7$ \\
\hline Female & 56.2 & 55 & 55.2 & 55.5 & 59.2 \\
\hline $\mathrm{BMI} \mathrm{kg} \cdot \mathrm{m}^{-2}$ & 28.3 & 27.1 & 29.1 & 29.1 & 29.9 \\
\hline Waist circumference cm & 97.6 & 93.8 & 96.1 & 99.8 & 102 \\
\hline Height $\mathrm{cm}$ & 166.8 & 166.9 & 167.2 & 166.4 & 165.6 \\
\hline eGFR $\mathrm{mL} \cdot \mathrm{min}^{-1} \cdot 1.73 \mathrm{~m}^{-2}$ & & 82.9 & 80.4 & 80.4 & 81.2 \\
\hline \multicolumn{6}{|l|}{ Race } \\
\hline White & 49.2 & 30 & 55.1 & 55.7 & 49.1 \\
\hline Chinese American & 12.2 & 24.2 & 13.2 & 6 & 3.3 \\
\hline Black, African American & 18 & 30.8 & 13 & 13.2 & 17.3 \\
\hline Hispanic & 20.8 & 14.7 & 18.7 & 25.1 & 30.2 \\
\hline \multicolumn{6}{|l|}{ Smoking } \\
\hline Ever-smokers & 45.7 & 47.7 & 45.3 & 49.6 & 60.9 \\
\hline Current smokers & 15.1 & 7.5 & 8.3 & 12.1 & 28.8 \\
\hline \multicolumn{6}{|l|}{ Site } \\
\hline Wake Forest & 16.8 & 13.4 & 18.9 & 14.4 & 17.3 \\
\hline Columbia & 16.7 & 17.9 & 12.6 & 16.3 & 19 \\
\hline Johns Hopkins & 12.7 & 15.6 & 11.5 & 12.9 & 12.1 \\
\hline UMN & 15.8 & 6.6 & 14.1 & 14.34 & 24.8 \\
\hline Northwestern & 15.5 & 19.5 & 18.4 & 17.2 & 8.3 \\
\hline UCLA & 22.5 & 27 & 24.5 & 22.9 & 18.4 \\
\hline
\end{tabular}

Data presented as mean \pm SD or $\%$, unless otherwise stated. All parameters were collected at MESA baseline visit in years 2000-2002. BMI: body mass index; eGFR: estimated glomerular filtration rate; UMN: Univeristy of Minnesota; UCLA: University of California, Los Angeles.

2.94\% (95\% CI 1.80-4.07\%, p<0.001), 1.24\% (95\% CI $0.14-2.35 \%, \mathrm{p}=0.03$ ) and $1.58 \%$ (CI $0.92-2.23 \%$, $\mathrm{p}<0.001$ ) increase in HAA, respectively (table 2, figure 1a-c). There was no evidence of effect modification by smoking status, age or BMI on the association between these adhesion molecules and HAA ( $\mathrm{p}$ for interaction $>0.08$; supplementary tables S6-S8). After correction for multiple comparisons, the p-values for sICAM-1, sVCAM-1 and P-selectin remained significant. Findings were consistent in a less adjusted model (supplementary table S9). The effect estimate for sICAM-1 was similar when the analysis was limited to individuals with the AA genotype of SNP rs5491 (supplementary table S10). There were no significant associations of E-selectin or L-selectin with HAA.

\section{Interstitial lung abnormalities}

In fully adjusted models, higher levels of sICAM-1 were associated with a greater prevalence of ILAs on CT scan. Each standard deviation increment of sICAM-1 was associated with 1.39 (95\% CI 1.13-1.71, $\mathrm{p}=0.002$ ) higher odds of ILA (table 2, figure $1 \mathrm{~d}-\mathrm{e}$ ). Unadjusted analyses by ILA subtype are shown in supplementary table S11. Each standard deviation increment of sICAM-1 was associated with 1.39 (95\% CI 1.14-1.70, $\mathrm{p}=0.001$ ) higher odds of fibrotic ILA subtypes, as defined by the presence of reticular abnormalities, traction bronchiectasis or honeycombing. There was no evidence of effect modification by smoking status, age or BMI on the association between sICAM-1 and ILA ( $\mathrm{p}$ for interaction $>0.05$; supplementary tables S6-8). When corrected for multiple comparisons, the p-value remained significant. Results for sICAM-1 were similar in analyses restricted to individuals with AA genotype of SNP rs5491 (supplementary table S10). There were no significant associations of sVCAM-1, P-selectin, E-selectin or L-selectin with ILA (table 2, figure 2f). Each standard deviation increment of VCAM-1 was associated with 1.34 higher odds of fibrotic ILA (supplementary table S11).

\section{Lung function}

In fully adjusted models, higher levels of sICAM-1 and P-selectin were associated with a lower FVC. Each standard deviation increment in sICAM-1 and P-selectin was associated with a $44.1 \mathrm{~mL}$ (95\% CI 12.4-75.9, $\mathrm{p}=0.006$ ) and $28.5 \mathrm{~mL}$ (95\% CI 8.0-49.1, p=0.007) lower FVC, respectively (table 2, figure 1g-i). 
TABLE 2 Associations of circulating adhesion molecules with high attenuation areas (HAA), interstitial lung abnormalities (ILA) and forced vital capacity (FVC)

\begin{tabular}{|c|c|c|c|c|c|c|c|c|c|c|}
\hline & sICAM-1 & p-value & sVCAM-1 & p-value & P-selectin & p-value & E-selectin & p-value & L-selectin & $\mathrm{p}$-value \\
\hline \multicolumn{11}{|l|}{ HAA } \\
\hline Participants & 2226 & & 2187 & & 5498 & & 864 & & 2187 & \\
\hline \multicolumn{11}{|l|}{ Change $\%(95 \% \mathrm{Cl})^{\#}$} \\
\hline Unadjusted & $2.87(1.38-4.36)$ & $<0.001$ & $1.82(0.34-3.31)$ & 0.02 & $1.79(0.87-2.72)$ & $<0.001$ & $4.87(2.51-7.23)$ & 0.01 & $-0.96(-2.45-0.53)$ & 0.21 \\
\hline Adjusted & $2.94(1.80-4.07)$ & $<0.001$ & $1.24(0.14-2.35)$ & 0.03 & $1.58(0.92-2.23)$ & $<0.001$ & $0.64(-1.21-2.42)$ & 0.51 & $0.40(-0.70-1.49)$ & 0.48 \\
\hline \multicolumn{11}{|l|}{ ILA } \\
\hline Participants & 1028 & & 1008 & & 2239 & & 507 & & 1008 & \\
\hline \multicolumn{11}{|l|}{ OR $(95 \% \mathrm{CI})^{\pi}$} \\
\hline Unadjusted & $1.41(1.18-1.67)$ & $<0.001$ & $1.33(1.13-1.56)$ & 0.001 & $1.10(0.97-1.24)$ & 0.14 & $0.88(0.67-1.17)$ & 0.37 & $0.91(0.75-0.09)$ & 0.30 \\
\hline Adjusted & $1.39(1.13-1.71)$ & 0.002 & $1.20(0.99-1.45)$ & 0.07 & $1.08(0.94-1.24)$ & 0.27 & $0.96(0.79-1.30)$ & 0.78 & $0.93(0.75-1.15)$ & 0.49 \\
\hline \multicolumn{11}{|l|}{ FVC } \\
\hline Participants & 1549 & & 1680 & & 3449 & & 808 & & 1688 & \\
\hline \multicolumn{11}{|l|}{ Change $\mathrm{mL}(95 \% \mathrm{Cl})^{\pi}$} \\
\hline Unadjusted & $-47.8(-94.5--0.6)$ & 0.05 & $-77.8(-120.0--35.6)$ & 0.001 & 60.7 (30.0-91.5) & $<0.001$ & $-36.81(-102.6-28.9)$ & 0.27 & $-9.47(-51.8-32.9)$ & 0.66 \\
\hline Adjusted & $-44.1(-75.9--12.4)$ & 0.006 & $-29.1(-57.7--0.4)$ & $0.047^{+}$ & $-28.5(-49.1--8.0)$ & 0.007 & $-43.9(-87.6--0.1)$ & $0.049^{+}$ & $10.6(-17.7-38.9)$ & 0.46 \\
\hline $\begin{array}{l}\text { Data are presented as } \mathrm{n} \\
\text { vascular adhesion mole } \\
\text { pack-year smoking hist } \\
\text { status, eGFR, BMI and } \mathrm{s}\end{array}$ & $\begin{array}{l}\text { unless otherwise state } \\
\text { le. }{ }^{\#} \text { : adjusted for age } \\
y \text { educational attainm } \\
\text {; }^{+}: \text {-value no longer }\end{array}$ & $\begin{array}{l}\text { d. Effect e } \\
\text { sex, bod } \\
\text { hent, study } \\
\text { significan }\end{array}$ & $\begin{array}{l}\text { stimates are per standa } \\
\text { y mass index (BMI), wai } \\
\text { y site/scanner, radiation } \\
\text { tt after Benjamini-Hochb }\end{array}$ & $\begin{array}{l}\text { rd deviati } \\
\text { st circumf } \\
\text { dose, pe } \\
\text { oerg corre }\end{array}$ & $\begin{array}{l}\text { on of each adhesion } r \\
\text { ference, race/ethnicit) } \\
\text { rcentage emphysema } \\
\text { ection for multiple cor }\end{array}$ & $\begin{array}{l}\text { nolecule. } \\
\text {, estimate }\end{array}$ & $\begin{array}{l}\text { ICAM: soluble intracell } \\
\text { d glomerular filtration } \\
\text { imaged lung volume; }\end{array}$ & $\begin{array}{l}\text { ular adhes } \\
\text { rate leGFR } \\
\text { ๆ: adjuste }\end{array}$ & $\begin{array}{l}\text { ion molecule; sVCAM } \\
\text { d, current smoking st } \\
\text { d for age, sex, race, }\end{array}$ & $\begin{array}{l}\text { 1: soluble } \\
\text { tatus and } \\
\text { smoking }\end{array}$ \\
\hline
\end{tabular}



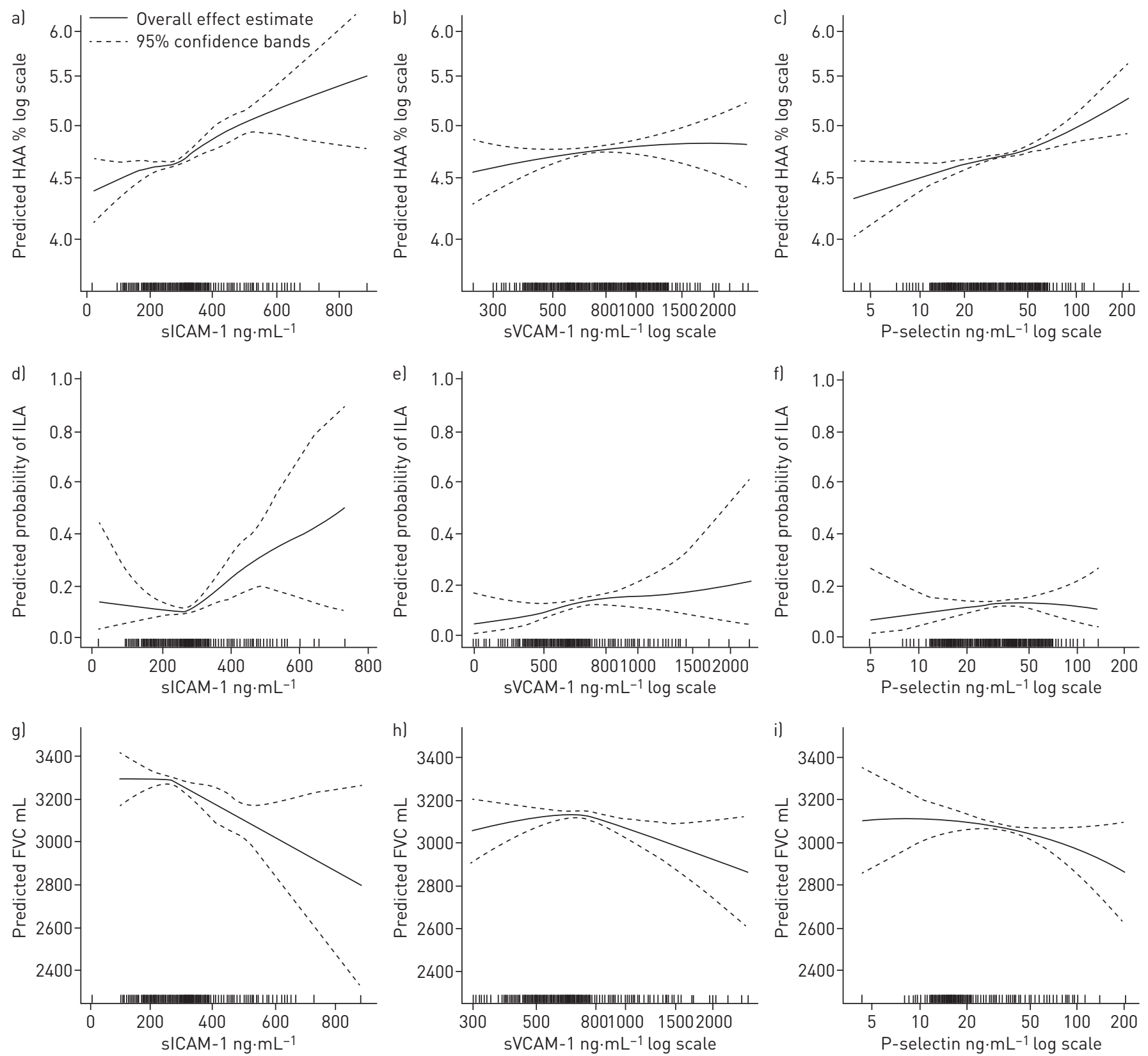

FIGURE 1 Continuous associations of adhesion molecules soluble intracellular adhesion molecule (sICAM)-1, soluble vascular adhesion molecule (sVCAM)-1 and P-selectin with high attenuation areas (HAA) (a-c, respectively), interstitial lung abnormalities (ILA) (d-f, respectively) and forced vital capacity (FVC) ( $g-i$, respectively). HAA models (a-c) are adjusted for age, sex, body mass index (BMI), waist circumference, height, race/ ethnicity, current smoking status and pack-year smoking history, educational attainment, glomerular filtration rate, study site/scanner, radiation dose, percentage emphysema and total imaged lung volume. ILA and FVC models ( $d$-i) are adjusted for age, sex, race, smoking status, BMI and study site. Each vertical hashmark in the rug plot along the $\mathrm{x}$-axis represents one study participant.

The association of sICAM-1 with FVC was modified by smoking status, with stronger effect estimates among ever-smokers ( $\mathrm{p}$ for interaction 0.01 ; supplementary table S6). There was no evidence of effect modification by age or BMI ( $\mathrm{p}$ for interaction $>0.05$; supplementary tables $\mathrm{S} 7$ and $\mathrm{S} 8$ ). There were significant associations of sVCAM-1 and E-selectin with FVC among ever-smokers, although the p-values for interaction were not significant (0.08 and 0.28 , respectively; supplementary table S6). Association between adhesion molecules with percentage predicted FVC are shown in supplementary table S12. Results were consistent when participants with an obstructive ventilator defect (forced expiratory volume in $1 \mathrm{~s}$ $(\mathrm{FEV} 1) / \mathrm{FVC}$ ratio $<70 \%$ ) were excluded from the analyses and among participants with the AA genotype of SNP rs5491 (supplementary tables S9 and S13). There was no significant association between L-selectin and FVC. 

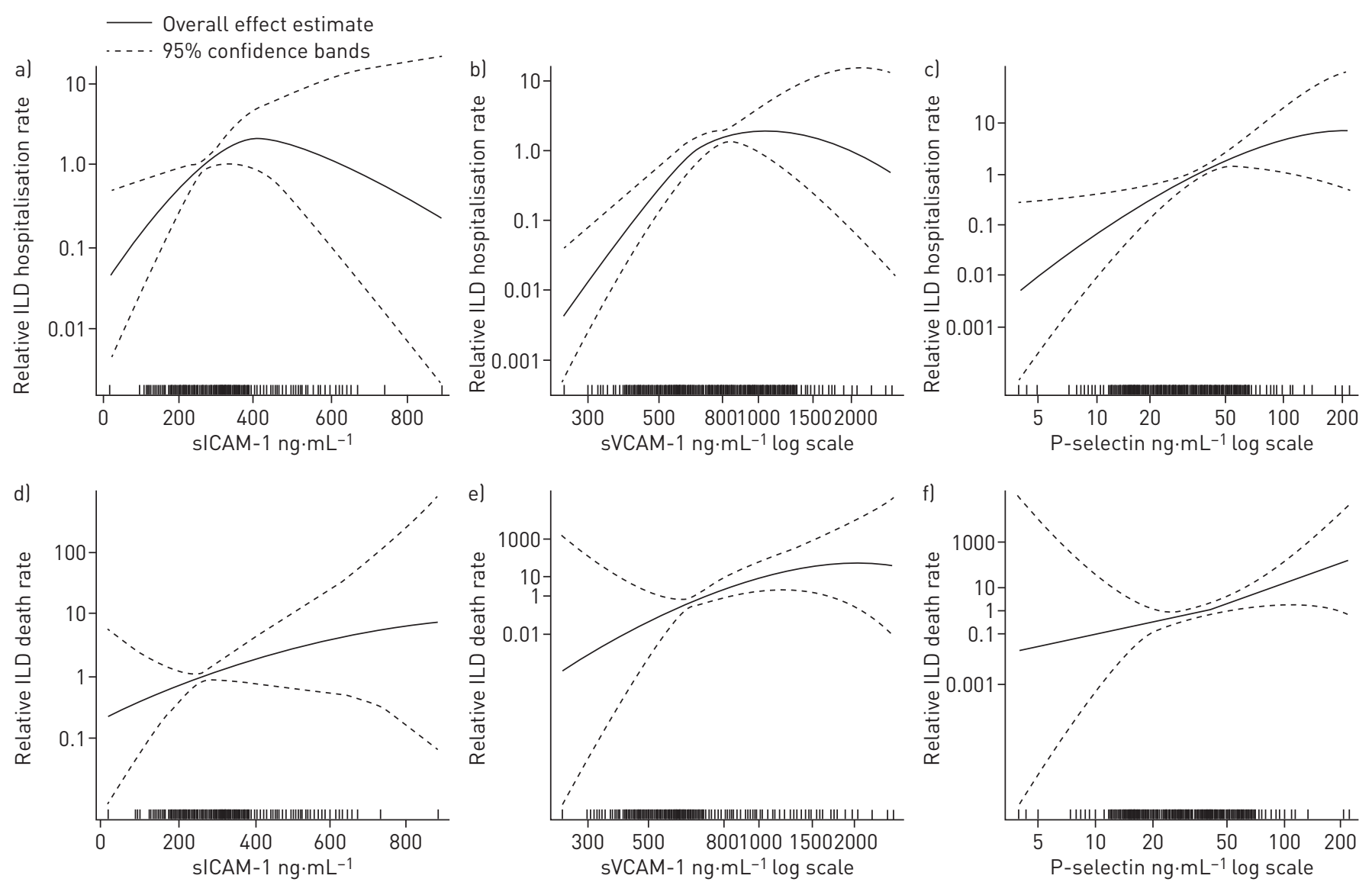

FIGURE 2 Continuous associations of adhesion molecules soluble intracellular adhesion molecule (sICAM)-1, soluble vascular adhesion molecule (sVCAM)-1 and P-selectin with interstitial lung disease (ILD) hospitalisation (a-c, respectively) and death due to ILD (d-f, respectively). Models adjusted for generalised propensity score 2, which included age, sex, race/ethnicity, smoking status, cigarette pack-years, body mass index, waist circumference, height, educational attainment, study site/scanner, glomerular filtration rate, radiation dose, alcohol use, total intentional exercise (metabolic equivalent min $\cdot$ week $^{-1}$ ), coronary artery calcium, diabetes medication use, insulin use, fasting glucose, hypertension, antihypertensive medication use, systolic and diastolic blood pressures, cholesterol medication use, total and high-density lipoprotein cholesterol levels, C-reactive protein, D-dimer and cancer history. Each vertical hashmark in the rug plot along the x-axis represents one study participant.

\section{ILD hospitalisations and death}

In fully adjusted models, higher levels of sICAM-1, sVCAM-1 and P-selectin were all associated with greater rate of respiratory hospitalisations among those with ILD, as well as hospitalisations due to ILD. Each standard deviation increment of sICAM-1, sVCAM-1 and P-selectin was associated with a hazard ratio of 1.36 (95\% CI 1.03-1.80, p=0.03), 1.40 (95\% CI 1.07-1.85, p=0.02) and 2.03 (95\% CI 1.16-3.55, $\mathrm{p}=0.01$ ), respectively, for ILD hospitalisation (table 3 , figure $2 \mathrm{a}-\mathrm{c}$ ). In fully adjusted models, both sVCAM-1 and P-selectin were associated with increased risk of death due to respiratory causes and ILD deaths. Each standard deviation increment in SVCAM-1 and P-selectin was associated with a hazard ratio of 2.15 (95\% CI 1.26-3.64, p=0.005) and 3.61 (95\% CI 1.54-8.46, p=0.003), respectively, for ILD death (table 3 , figure $2 \mathrm{e}-\mathrm{f}$ ). There was a trend toward an increased risk for both respiratory and ILD mortality in participants with higher sICAM-1 levels, but the association did not reach statistical significance (table 3 , figure $2 \mathrm{~d}$ ). There were no significant associations of E-selectin and L-selectin with ILD events.

\section{Discussion}

We found that higher levels of circulating sICAM-1, sVCAM-1 and P-selectin were each independently associated with CT measures of subclinical ILD, lower FVC, increased rate of adjudicated ILD hospitalisations and ILD death among community-dwelling adults. E-selectin was associated with lower FVC and respiratory mortality, but not our other outcome measures. These findings strongly support roles for circulating adhesion molecules sICAM-1, sVCAM-1 and P-selectin in the development of ILD and offer important insights into the biological processes behind previous studies showing association of HAA and ILA with disease progression and mortality $[9,25]$.

ICAM-1 is essential for leukocyte diapedesis, which may be important in the earliest stages of pulmonary fibrosis $[10,12]$. In the healthy lung, ICAM-1 is expressed in alveolar macrophages and, at low levels, in 


\begin{tabular}{|c|c|c|c|c|c|c|c|c|c|c|}
\hline & SICAM-1 & p-value & sVCAM-1 & p-value & P-selectin & p-value & E-selectin & p-value & L-selectin & p-value \\
\hline \multicolumn{11}{|c|}{ Respiratory hospitalisation with concomitant ILD } \\
\hline Events & 20 & & 18 & & 77 & & 9 & & 18 & \\
\hline Person-years & 30295 & & 28014 & & 70176 & & 11457 & & 28014 & \\
\hline Rate per 10000 person-years $(95 \% \mathrm{CI})$ & $6.6(4.3-10.2)$ & & $6.4(4.0-10.2)$ & & $11.0(8.8-13.7)$ & & $7.9(4.1-15.1)$ & & $6.4(4.0-10.2)$ & \\
\hline \multicolumn{11}{|l|}{ Hazard ratio $(95 \% \mathrm{CI})$} \\
\hline Unadjusted & $1.75(1.46-2.10)$ & $<0.001$ & $1.56(1.27-1.90)$ & $<0.001$ & $1.73(1.23-2.43)$ & 0.002 & $1.39(1.02-1.89)$ & 0.04 & $1.09(0.67-1.71)$ & 0.78 \\
\hline Adjusted: model $1^{\#}$ & $1.36(1.13-1.63)$ & 0.001 & $1.62(1.21-2.16)$ & 0.001 & $1.84(1.24-2.72)$ & 0.002 & $1.29(0.71-2.35)$ & 0.40 & $1.09(0.65-1.84)$ & 0.75 \\
\hline Adjusted: model 2" & $1.34(1.09-1.66)$ & 0.006 & $1.68(1.25-2.27)$ & $<0.001$ & $2.07(1.25-3.43)$ & 0.005 & $1.42(0.75-2.70)$ & 0.29 & $1.09(0.66-1.80)$ & 0.75 \\
\hline \multicolumn{11}{|l|}{ ILD hospitalisation } \\
\hline Events & 12 & & 11 & & 44 & & 8 & & 11 & \\
\hline Person-years & 30295 & & 28014 & & 70176 & & 11457 & & 28014 & \\
\hline Rate per 10000 person-years $(95 \% \mathrm{CI})$ & $4.0(2.3-7.0)$ & & $3.9(2.2-7.1)$ & & $6.3(4.7-8.4)$ & & $7.0(3.5-14.0)$ & & $3.9(2.2-7.1)$ & \\
\hline \multicolumn{11}{|l|}{ Hazard ratio $(95 \% \mathrm{CI})$} \\
\hline Unadjusted & $1.70(1.32-2.21)$ & $<0.001$ & $1.57(1.21-2.05)$ & $<0.001$ & $1.95(1.32-2.88)$ & $<0.001$ & $1.34(0.94-1.91)$ & 0.11 & $1.10(0.52-2.33)$ & 0.80 \\
\hline Adjusted: model $1^{\#}$ & $1.30(1.00-1.70)$ & $0.047^{+}$ & $1.53(1.24-1.89)$ & $<0.001$ & $1.87(1.19-2.92)$ & 0.007 & $1.25(0.65-2.41)$ & 0.51 & $1.03(0.58-1.84)$ & 0.91 \\
\hline Adjusted: model 2" & $1.36(1.03-1.80)$ & 0.03 & $1.40(1.07-1.85)$ & 0.02 & $2.03(1.16-3.55)$ & 0.01 & $1.30(0.72-2.36)$ & 0.38 & $1.05(0.56-1.97)$ & 0.87 \\
\hline \multicolumn{11}{|l|}{ Respiratory mortality } \\
\hline Events & 28 & & 25 & & 63 & & 7 & & 25 & \\
\hline Person-years & 30295 & & 28014 & & 70176 & & 11457 & & 28014 & \\
\hline Rate per 10000 person-years $(95 \% \mathrm{Cl})$ & $9.2(6.4-13.4)$ & & $8.9(6.0-13.2)$ & & $9.0(7.0-11.5)$ & & $6.1(2.9-12.8)$ & & $8.9(6.0-13.2)$ & \\
\hline \multicolumn{11}{|l|}{ Hazard ratio $(95 \% \mathrm{Cl})$} \\
\hline Unadjusted & $1.43(1.08-1.91)$ & $0.01^{+}$ & $1.71(1.42-2.06)$ & $<0.001$ & $1.48(1.14-1.92)$ & 0.003 & $1.78(1.13-2.81)$ & $0.01^{+}$ & $0.85(0.56-1.29)$ & 0.43 \\
\hline Adjusted: model $1^{\#}$ & $1.25(0.94-1.65)$ & 0.13 & $1.80(1.38-2.36)$ & $<0.001$ & $1.49(1.12-1.98)$ & 0.006 & $1.95(0.97-3.94)$ & 0.06 & $0.85(0.59-1.23)$ & 0.39 \\
\hline Adjusted: model $2^{\pi}$ & $1.29(0.96-1.72)$ & 0.09 & $2.06(1.56-2.72)$ & $<0.001$ & $1.54(1.15-2.08)$ & 0.004 & $1.95(1.04-3.65)$ & $0.04^{+}$ & $0.84(0.58-1.21)$ & 0.35 \\
\hline \multicolumn{11}{|l|}{ ILD mortality } \\
\hline Events & 9 & & 7 & & 16 & & 3 & & 7 & \\
\hline Person-years & 30295 & & 28014 & & 70176 & & 11457 & & 28014 & \\
\hline Rate per 10000 person-years $(95 \% \mathrm{CI})$ & $3.0(1.5-5.7)$ & & $2.5(1.2-5.2)$ & & $2.3(1.4-3.7)$ & & $2.6(0.8-8.1)$ & & $2.5(1.2-5.2)$ & \\
\hline \multicolumn{11}{|l|}{ Hazard ratio $(95 \% \mathrm{Cl})$} \\
\hline Unadjusted & $1.83(1.31-2.55)$ & $<0.001$ & $1.71(1.21-2.43)$ & 0.003 & $2.04(1.25-3.33)$ & 0.004 & $1.35(0.56-3.26)$ & 0.51 & $1.47(0.76-2.87)$ & 0.25 \\
\hline Adjusted: model $1^{\#}$ & $1.41(0.93-2.14)$ & 0.11 & $2.02(1.19-3.41)$ & 0.009 & $3.30(1.40-7.80)$ & 0.006 & $1.28(0.45-3.65)$ & 0.64 & $1.29(0.67-2.50)$ & 0.45 \\
\hline Adjusted: model $2^{\uparrow}$ & $1.39(0.94-2.07)$ & 0.10 & $2.15(1.26-3.64)$ & 0.005 & $3.61(1.54-8.46)$ & 0.003 & $1.36(0.52-3.58)$ & 0.53 & $1.29(0.66-2.53)$ & 0.45 \\
\hline \multicolumn{11}{|c|}{$\begin{array}{l}\text { Data are presented as } \mathrm{n} \text {, unless otherwise stated. Effect estimates are per standard deviation of each adhesion molecule. sICAM: soluble intracellular adhesion molecule; sVCAM: soluble } \\
\text { vascular adhesion molecule. \#: adjusted for generalised propensity score 1, which includes age, sex, race/ethnicity, smoking status, cigarette pack-years, body mass index (BMI), waist } \\
\text { circumference, height, educational attainment, study site and glomerular filtration rate (GFR); }{ }^{\natural}: \text { adjusted for generalised propensity score } 2 \text {, which includes age, sex, race/ethnicity, } \\
\text { smoking status, cigarette pack-years, BMI, waist circumference, height, educational attainment, study site, GFR, percentage emphysema on computed tomography, alcohol use, total } \\
\text { intentional exercise (metabolic equivalent min.week }{ }^{-1} \text { ), coronary artery calcium, diabetes medication use, insulin use, fasting glucose, hypertension, antihypertensive medication use, } \\
\text { systolic and diastolic blood pressures, cholesterol medication use, total and high-density lipoprotein cholesterol levels, C-reactive protein, D-dimer and cancer history; }{ }^{+} \text {: p-value no } \\
\text { longer significant after correcting for multiple comparisons. }\end{array}$} \\
\hline
\end{tabular}


type II alveolar epithelial cells (AECs) and capillary endothelial cells [26, 27]. In mouse models, administration of bleomycin leads to upregulation of ICAM-1 expression on macrophages and type II AECs [10]. In humans with IPF, plasma concentrations of sICAM-1 are associated with poor progression-free and overall survival [12]. Ours is the first study to demonstrate associations between circulating levels of sICAM-1 and subclinical ILD. These findings may point to a role for sICAM-1 as a serum marker of type II AEC injury, or perhaps of activated macrophages, both of which have been implicated in the pathobiology of early ILD. AARON et al. [28] showed that higher sICAM-1 levels are associated with greater progression of emphysema, but not with longitudinal change in lung function, as measured by FEV1 or FEV1/FVC ratio. We demonstrate an association of sICAM-1 with both CT measures of early ILD and lower FVC, even after adjusting for emphysema. Further studies are needed to determine whether sICAM-1 is directly involved in the pathobiology of early ILD, or is a biomarker of the disease process.

VCAM-1 is expressed within the vascular endothelium and functions similarly to ICAM-1 with regard to leukocyte recruitment [27]. Exposure to radiation increases endothelial expression of endothelial VCAM-1 in mouse models of pulmonary fibrosis [29]. In humans with IPF, both lung tissue expression of VCAM-1 and plasma levels of sVCAM-1 are significantly increased compared to controls [30]. In addition, plasma levels of sVCAM-1 have been shown to be predictive of mortality [12]. VCAM-1 is densely expressed in fibrotic foci of IPF lungs and its protein and mRNA levels significantly increase when human lung fibroblasts are treated with transforming growth factor (TGF)- $\beta$ [30]. Therefore, VCAM-1 may play a role in fibroblast proliferation in addition to leukocyte recruitment.

Ours is the first study to implicate P-selectin in the development and progression of ILD in humans. P-selectin is expressed on the surface of both activated endothelial cells and platelets [31]. When platelets adhere to endothelium at a site of injury, P-selectin on the surface of platelets binds neutrophils, monocytes and lymphocytes and slows their flow [31]. Activated platelets, bound to the capillary endothelium, also induce fibrin binding and upregulate endothelial expression of ICAM-1 and VCAM-1 [32]. Little is known about the role of P-selectin in the development of fibrosis, but there are substantial data supporting the role of platelets and P-selectin in acute lung injury, which is particularly relevant given that repetitive injury to alveolar membrane has been implicated in the development of ILD, and subclinical ILD has been linked to increased risk of acute respiratory distress syndrome [33]. In animal models of acute lung injury, plasma levels of P-selectin correlated with the extent of lung inflammation after infusion of lipopolysaccharide, and an increase in platelet-derived P-selectin led to downstream ICAM-1 expression and neutrophil activation [34, 35]. In patients with acute respiratory distress syndrome, plasma P-selectin levels are associated with both higher lung injury scores and increased mortality [2, 15]. Our findings of an association of plasma P-selectin levels with subclinical ILD may therefore reflect increased platelet activity, fibroblast activation and upregulation of other adhesion molecules in early ILD.

We found that E-selectin was associated with lower FVC and respiratory mortality, but not with other measures of subclinical ILD, and that L-selectin was not associated with any of our outcomes. E-selectin is expressed on endothelial cells and has been shown to be upregulated in bleomycin-induced endothelial injury [36]. Given the relatively low sample size of the E-selectin analyses, it is possible that our study was not powered to detect an association between E-selectin and subclinical ILD. Alternatively, it is possible that E-selectin plays a role in more advanced stage of ILD, especially given prior data, which showed that in IPF lungs, E-selectin expression is restricted to areas of honeycombing [37]. L-selectin is constitutively expressed on the surface of leukocytes and its expression is not affected by endothelial perturbation, which may be one explanation for our null findings [38].

The association between sICAM-1 and lung function was modified by smoking status. Smoking is a well-known risk factor for development of IPF and tobacco use is associated with both decreased FVC as well as increased HAA in the MESA cohort [5]. Underlying mechanisms may include oxidative stress to alveolar epithelium, and the release of profibrotic factors such as TGF- $\beta$ from pulmonary fibroblasts [39]. SCHABERG et al. [40] demonstrated that smoking upregulates endothelial expression of ICAM-1 within the peripheral pulmonary vasculature, suggesting that smoking may lead to recruitment of leukocytes. Thus, while higher sICAM-1 levels are associated with a decrease in FVC when adjusted for smoking status, smoking and sICAM-1 may interact to jointly cause additional oxidative stress to the epithelium and recruitment of leukocytes.

There were several limitations to our study. First, all adhesion molecules were measured in serum or plasma rather than lung tissue or single lung cells, and thus may not fully reflect pathobiological changes in the lung. Moreover, measurements were performed on stored samples and were not run in duplicate. Therefore, there is some concern about protein degradation or even estimate inflation. However, we believe that the relative differences are important even if the absolute values are not completely accurate. Second, 
adhesion molecules were measured at a single time point, limiting our ability to study the associations of longitudinal trends in these adhesion molecules with both subclinical and clinical measures of ILD. Third, analyses of clinical ILD events are limited by low event rates. Fourth, multiple comparisons increase the chances of type I error while varying sample sizes limits our ability to compare results between biomarkers. Finally, while we adjusted for a number of demographic and clinical variables in our models, there remains the potential that residual confounding may affect some or all of our results.

In summary, we found that circulating adhesion molecules sICAM-1, sVCAM-1 and P-selectin are linked to CT measures of early lung injury, inflammation and fibrosis, lower FVC and clinical ILD events in community-dwelling adults, supporting their role as potential peripheral blood biomarkers of subclinical ILD. These findings suggest that sICAM-1, sVCAM-1 and P-selectin may be important in the pathogenesis of ILD, and warrant future studies of the role played by these adhesion molecules in the development and progression of pulmonary fibrosis.

Support statement: This work was funded by the US Department of Health and Human Services, National Institutes of Health (HHSN268201500003I, N01-HC-95159, N01-HC-95160, N01-HC-95161, N01-HC-95162, N01-HC-95163, N01-HC-95164, N01-HC-95165, N01-HC-95166, N01-HC-95167, N01-HC-95168, N01-HC-95169). Funding information for this article has been deposited with the Crossref Funder Registry.

Conflict of interest: C.F. McGroder has nothing to disclose. C.P. Aaron reports grants from NIH, during the conduct of the study; grants from Alpha-1 Foundation and Stony-Wold Herbert Fund, Inc., personal fees from Lancet Respiratory Medicine, outside the submitted work. S.J. Bielinski reports a grant from NIH, during the conduct of the study. S.M. Kawut reports grants from NIH, during the conduct of the study; non-financial (travel) support from ATS and the Pulmonary Hypertension Association, grants for education from Actelion, United Therapeutics, Gilead, Lung Biotech, Bayer and Mallinkrodt, grants and non-financial support from Cardiovascular Medical Research and Education Fund, outside the submitted work; and has served in an advisory capacity (for grant review and other purposes) for United Therapeutics, Akros Pharmaceuticals, GlaxoSmithKline and Complexa, Inc. without financial support or in-kind benefits. R.P. Tracy reports grants from NIH, during the conduct of the study. G. Raghu has nothing to disclose. R.G. Barr has nothing to disclose. D.J. Lederer reports personal fees for steering committee work from Roche and Galapagos, personal fees for advisory board work from Sanofi Genzyme and Philips Respironics, grants and personal fees for advisory board work and consultancy from Fibrogen, Global Blood Therapeutics and Boehringer Ingelheim, and fees for consultancy paid directly to Columbia University from Pulmonary Fibrosis Foundation, outside the submitted work; since May 2018, D.J. Lederer no longer receives fees from industry sources. A.J. Podolanczuk has nothing to disclose.

\section{References}

1 Rosas IO, Dellaripa PF, Lederer DJ, et al. Interstitial lung disease: NHLBI Workshop on the Primary Prevention of Chronic Lung Diseases. Ann Am Thorac Soc 2014; 11: Suppl. 3, S169-S177.

2 Bone RC, Francis PB, Pierce AK. Intravascular coagulation associated with the adult respiratory distress syndrome. Am J Med 1976; 61: 585-589.

3 Selman M, Thannickal VJ, Pardo A, et al. Idiopathic pulmonary fibrosis: pathogenesis and therapeutic approaches. Drugs 2004; 64: 405-430.

4 Raghu G, Collard HR, Egan JJ, et al. An official ATS/ERS/JRS/ALAT statement: idiopathic pulmonary fibrosis: evidence-based guidelines for diagnosis and management. Am J Respir Crit Care Med 2011; 183: 788-824.

5 Lederer DJ, Enright PL, Kawut SM, et al. Cigarette smoking is associated with subclinical parenchymal lung disease: the Multi-Ethnic Study of Atherosclerosis (MESA)-lung study. Am J Respir Crit Care Med 2009; 180: 407-414.

6 Podolanczuk AJ, Oelsner EC, Barr RG, et al. High attenuation areas on chest computed tomography in community-dwelling adults: the MESA study. Eur Respir J 2016; 48: 1442-1452.

7 Washko GR, Hunninghake GM, Fernandez IE, et al. Lung volumes and emphysema in smokers with interstitial lung abnormalities. N Engl J Med 2011; 364: 897-906.

8 Putman RK, Hatabu H, Araki T, et al. Association between interstitial lung abnormalities and all-cause mortality. JAMA 2016; 315: 672-681.

9 Podolanczuk AJ, Oelsner EC, Barr RG, et al. High-attenuation areas on chest computed tomography and clinical respiratory outcomes in community-dwelling adults. Am J Respir Crit Care Med 2017; 196: 1434-1442.

10 Fernandez IE, Amarie OV, Mutze K, et al. Systematic phenotyping and correlation of biomarkers with lung function and histology in lung fibrosis. Am J Physiol Lung Cell Mol Physiol 2016; 310: L919-L927.

11 Bless NM, Tojo SJ, Kawarai H, et al. Differing patterns of P-selectin expression in lung injury. Am J Pathol 1998; 153: 1113-1122.

12 Richards TJ, Kaminski N, Baribaud F, et al. Peripheral blood proteins predict mortality in idiopathic pulmonary fibrosis. Am J Respir Crit Care Med 2012; 185: 67-76.

13 Nakao A, Hasegawa Y, Tsuchiya Y, et al. Expression of cell adhesion molecules in the lungs of patients with idiopathic pulmonary fibrosis. Chest 1995; 108: 233-239.

14 Hamaguchi $\mathrm{Y}$, Nishizawa $\mathrm{Y}$, Yasui $\mathrm{M}$, et al. Intercellular adhesion molecule-1 and L-selectin regulate bleomycin-induced lung fibrosis. Am J Pathol 2002; 161: 1607-1618.

15 Sakamaki F, Ishizaka A, Handa M, et al. Soluble form of P-selectin in plasma is elevated in acute lung injury. Am J Respir Crit Care Med 1995; 151: 1821-1826.

16 Bild DE, Bluemke DA, Burke GL, et al. Multi-Ethnic Study of Atherosclerosis: objectives and design. Am J Epidemiol 2002; 156: 871-881.

17 Register TC, Burdon KP, Lenchik L, et al. Variability of serum soluble intercellular adhesion molecule-1 measurements attributable to a common polymorphism. Clin Chem 2004; 50: 2185-2187. 
18 Hoffman EA, Jiang R, Baumhauer H, et al. Reproducibility and validity of lung density measures from cardiac CT scans - the Multi-Ethnic Study of Atherosclerosis (MESA) Lung Study. Acad Radiol 2009; 16: 689-699.

19 Washko GR, Lynch DA, Matsuoka S, et al. Identification of early interstitial lung disease in smokers from the COPDGene Study. Acad Radiol 2010; 17: 48-53.

20 Hunninghake GM, Hatabu H, Okajima Y, et al. MUC5B promoter polymorphism and interstitial lung abnormalities. N Engl J Med 2013; 368: 2192-2200.

21 Miller MR, Hankinson J, Brusasco V, et al. Standardisation of spirometry. Eur Respir J 2005; 26: 319-338

22 Hankinson JL, Kawut SM, Shahar E, et al. Performance of American Thoracic Society-recommended spirometry reference values in a multiethnic sample of adults: the Multi-Ethnic Study of Atherosclerosis (MESA) lung study. Chest 2010; 137: 138-145.

23 Armstrong HF, Podolanczuk AJ, Barr RG, et al. Serum matrix metalloproteinase-7, respiratory symptoms, and mortality in community-dwelling adults. MESA (Multi-Ethnic Study of Atherosclerosis). Am J Respir Crit Care Med 2017; 196: 1311-1317.

24 Bielinski SJ, Berardi C, Decker PA, et al. P-selectin and subclinical and clinical atherosclerosis: the Multi-Ethnic Study of Atherosclerosis (MESA). Atherosclerosis 2015; 240: 3-9.

25 Miller ER, Putman RK, Vivero M, et al. Histopathology of interstitial lung abnormalities in the context of lung nodule resections. Am J Respir Crit Care Med 2018; 197: 955-958.

26 Paine R 3rd, Christensen P, Toews GB, et al. Regulation of alveolar epithelial cell ICAM-1 expression by cell shape and cell-cell interactions. Am J Physiol 1994; 266: L476-L484.

27 Osborn L. Leukocyte adhesion to endothelium in inflammation. Cell 1990; 62: 3-6.

28 Aaron CP, Schwartz JE, Bielinski SJ, et al. Intercellular adhesion molecule 1 and progression of percent emphysema: the MESA Lung Study. Respir Med 2015; 109: 255-264.

29 Epperly MW, Sikora CA, DeFilippi SJ, et al. Pulmonary irradiation-induced expression of VCAM-I and ICAM-I is decreased by manganese superoxide dismutase-plasmid/liposome (MnSOD-PL) gene therapy. Biol Blood Marrow Transplant 2002; 8: 175-187.

30 Agassandian M, Tedrow JR, Sembrat J, et al. VCAM-1 is a TGF- $\beta 1$ inducible gene upregulated in idiopathic pulmonary fibrosis. Cell Signal 2015; 27: 2467-2473.

31 Katz JN, Kolappa KP, Becker RC. Beyond thrombosis: the versatile platelet in critical illness. Chest 2011; 139 : 658-668.

32 Hammwöhner M, Ittenson A, Dierkes J, et al. Platelet expression of CD40/CD40 ligand and its relation to inflammatory markers and adhesion molecules in patients with atrial fibrillation. Exp Biol Med 2007; 232: 581-589.

33 Selman M, King TE, Pardo A, et al. Idiopathic pulmonary fibrosis: prevailing and evolving hypotheses about its pathogenesis and implications for therapy. Ann Intern Med 2001; 134: 136-151.

34 Hirose M, Murai T, Kawashima H. Elevation of rat plasma P-selectin in acute lung injury. Biochim Biophys Acta 2007; 1772: 382-389.

35 Zarbock A, Singbartl K, Ley K. Complete reversal of acid-induced acute lung injury by blocking of platelet-neutrophil aggregation. J Clin Invest 2006; 116: 3211-3219.

36 Liao JK. Linking endothelial dysfunction with endothelial cell activation. J Clin Invest 2013; 123: 540-541.

37 Hayashi S, Abe K, Matsuoka $\mathrm{H}$, et al. Increased level of soluble E-selectin in the serum from patients with idiopathic pulmonary fibrosis. Inflammation 2004; 28: 1-5.

38 Kelly M, Hwang JM, Kubes P. Modulating leukocyte recruitment in inflammation. J Allergy Clin Immunol 2007; 120: 3-10.

39 Nagy J, Demaster EG, Wittmann I, et al. Induction of endothelial cell injury by cigarette smoke. Endothelium 1997; 5: 251-263.

40 Schaberg T, Rau M, Oerter R, et al. Expression of adhesion molecules in peripheral pulmonary vessels from smokers and nonsmokers. Lung 1996; 174: 71-81. 